Eq. 3(c) must now be replaced by $-H_{t}$, and, since the left side of Eq. 3(c) is known at $t=t_{0}, H_{t}\left(t_{0}\right)$ is known throughout the field. A finite difference approximation then yields $H\left(t_{0}+\Delta t\right)$ throughout the field, so that the process may be repeated. Thus a step-by-step solution may be obtained for all values of $t$.

\title{
A NOTE ON THE PRINCIPAL FREQUENCY OF A TRIANGULAR MEMBRANE* '
}

\author{
By G. PÓLYA (Stanford University)
}

The principal frequency of a membrane of triangular shape is exactly known in two simple cases: for the $45^{\circ}, 45^{\circ}, 90^{\circ}$ and the $60^{\circ}, 60^{\circ}, 60^{\circ}$ triangles. ${ }^{2}$ As will be shown in this note, an exact solution of comparable simplicity exists also for the $30^{\circ}, 60^{\circ}, 90^{\circ}$ triangle-a result which, to the author's knowledge, has not been observed before.

The three lines the equations of which in rectangular coordinates $x, y$ are

$$
y=0, \quad x=a 3^{1 / 2} / 2, \quad y=x 3^{-1 / 2}
$$

delimit a triangle, one half of an equilateral triangle with side $a$. Define

$$
\begin{aligned}
u=\sin \pi( & \left(y-3^{-1 / 2} x\right) / a \cdot \sin \pi\left(y+3^{-1 / 2} x\right) / a \cdot \sin 2 \pi 3^{-1 / 2} x / a \\
\cdot & \sin \pi\left(y-3^{1 / 2} x\right) / 3 a \cdot \sin \pi\left(y+3^{1 / 2} x\right) / 3 a \cdot \sin 2 \pi y / 3 a
\end{aligned}
$$

By elementary transformations we find that

$$
\begin{aligned}
-32 u & =\cos 2 \pi\left(5 y+3^{1 / 2} x\right) / 3 a-\cos 2 \pi\left(4 y+3^{1 / 2} 2 x\right) / 3 a+\cos 2 \pi\left(y+3^{1 / 2} 3 x\right) / 3 a \\
& -\cos 2 \pi\left(5 y-3^{1 / 2} x\right) / 3 a+\cos 2 \pi\left(4 y-3^{1 / 2} 2 x\right) / 3 a-\cos 2 \pi\left(y-3^{1 / 2} 3 x\right) / 3 a .
\end{aligned}
$$

We see from (3) that $u$ satisfies the equation

$$
u_{x x}+u_{y y}+9^{-1} 112 \pi^{2} a^{-2} u=0,
$$

from (2) or (3) that $u$ vanishes along the lines (1), and from (2) that $u$ does not vanish in the interior of the triangle between the lines (1). Therefore, $u$ represents the principal mode.

The well known solution for the two other triangles mentioned at the beginning can be presented in a strictly analogous form. Any of these three triangles, repeated by successive reflections, covers the whole plane without overlapping and there are no further triangles of this kind. Therefore, there are no other triangles for which the solution can be presented in a comparably simple form as a function of $x, y$ without singularity in the whole plane. The last remarks indicate also the heuristic reasoning which led to the solution (2).

*Received April 17, 1950.

'Sponsored by the Office of Naval Research.

${ }^{2}$ Rayleigh, The theory of sound, Dover Publications, New York, 1945, §199. 